\title{
Autoimmune Pulmonary Alveolar Proteinosis Following Pulmonary Aspergillosis
}

\author{
Toru Arai ${ }^{1}$, Yoshikazu Inoue ${ }^{1}$, Masanori Akira ${ }^{1,2}$, Koh Nakata $^{3}$ and Masanori Kitaichi ${ }^{1,4,5}$
}

\begin{abstract}
A 59-year-old man, ex-smoker, was diagnosed with pulmonary aspergillosis according to chest radiography findings of a fungus ball and Aspergillus fumigatus detection in the bronchial lavage fluid. Two years after anti-fungal therapy, he was diagnosed with autoimmune pulmonary alveolar proteinosis (APAP) according to a crazy paving pattern in computed tomography scans of the chest, milky bronchoalveolar lavage effluent, and positive anti-granulocyte-macrophage colony-stimulating factor (GM-CSF) autoantibody in the serum. The patient ultimately died of respiratory failure caused by APAP four months after GM-CSF therapy commenced. Aspergillus infection may thus be associated with the onset and progression of APAP and tolerance to GM-CSF therapy.
\end{abstract}

Key words: pulmonary alveolar proteinosis, pulmonary aspergillosis, granulocyte-macrophage colonystimulating factor

(Intern Med 54: 3177-3180, 2015)

(DOI: 10.2169/internalmedicine.54.5034)

\section{Introduction}

Autoimmune pulmonary alveolar proteinosis (APAP) is characterized by the accumulation of phospholipids and surfactant protein in the alveolar spaces (1) due to macrophage dysfunction caused by anti-granulocyte-macrophage colonystimulating factor (GM-CSF) neutralizing antibody (2). Patients with APAP are immunocompromised because of functional disturbances of macrophages and neutrophils (3). Secondary infection $(4,5)$ with microorganisms, such as Nocardia (6), Mycobacterium (7), and Aspergillus $(8,9)$ species is also common in these patients. Infections have been reported to account for $18 \%$ of deaths in patients with APAP (4). We herein report a rare case of APAP in which Aspergillus infection may have caused the disease onset and/or progression of APAP.

\section{Case Report}

A 59-year-old man, ex-smoker, with a history of pulmonary tuberculosis complained of a dry cough for two months in November 2000. Chest radiography (Fig. 1A) and computed tomography (CT) (Fig. 2A, B) revealed a fungus ball in the pulmonary cavity of the left upper lobe that was caused by prior tuberculosis. According to these results, the patient was diagnosed with simple pulmonary aspergilloma (10). His serum was positive for anti-Aspergillus antibody. Aspergillus fumigatus was cultured from the bronchial lavage fluid collected from the left upper division of the bronchus. He was treated with itraconazole $(100 \mathrm{mg} /$ day $)$ and his cough temporally improved; however, 2 years after the commencement of therapy (Fig. 1B), ground-glass opacity with a crazy paving pattern along with the gradual enlargement of the fungus ball was observed in chest CT scans (Fig. 2C, D) and the serum $\beta$-D glucan level increased to $101 \mathrm{pg} / \mathrm{mL}$. Effluent obtained during the bronchoalveolar

\footnotetext{
${ }^{1}$ Department of Clinical Research Center, National Hospital Organization, Kinki-Chuo Chest Medical Center, Japan, ${ }^{2}$ Department of Radiology, National Hospital Organization, Kinki-Chuo Chest Medical Center, Japan, ${ }^{3}$ Bioscience Medical Research Center, Niigata University Medical and Dental Hospital, Japan, ${ }^{4}$ Department of Pathology, National Hospital Organization, Kinki-Chuo Chest Medical Center, Japan and ${ }^{5}$ Department of Pathology, National Hospital Organization Minami-Wakayama Medical Center, Japan Received for publication January 29, 2015; Accepted for publication April 7, 2015 Correspondence to Dr. Yoshikazu Inoue, giichi@kch.hosp.go.jp
} 


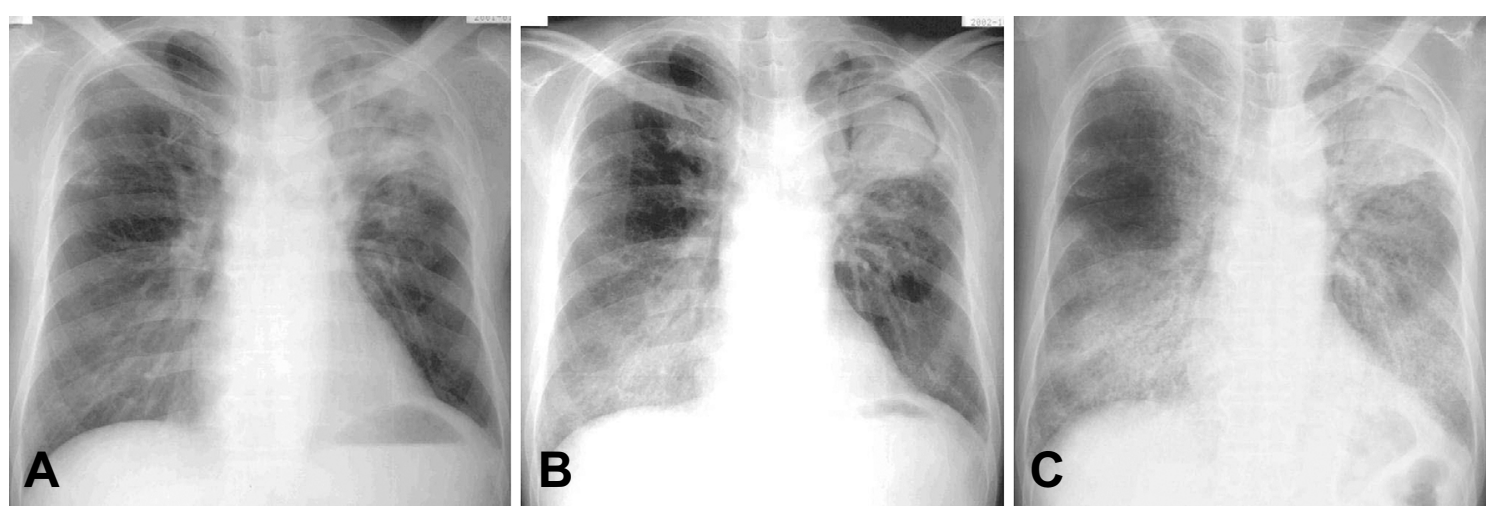

Figure 1. Changes in the chest radiographic findings. A fungus ball formed during previous tuberculosis appeared in the cavity of the left upper lobe (A). Two years after the diagnosis, the fields of the bilateral lower lung were found to exhibit ground-glass opacity (B), and complication of autoimmune pulmonary alveolar proteinosis (APAP) was diagnosed. APAP severity in the case increased 6 months after the diagnosis of $\operatorname{APAP}(C)$.
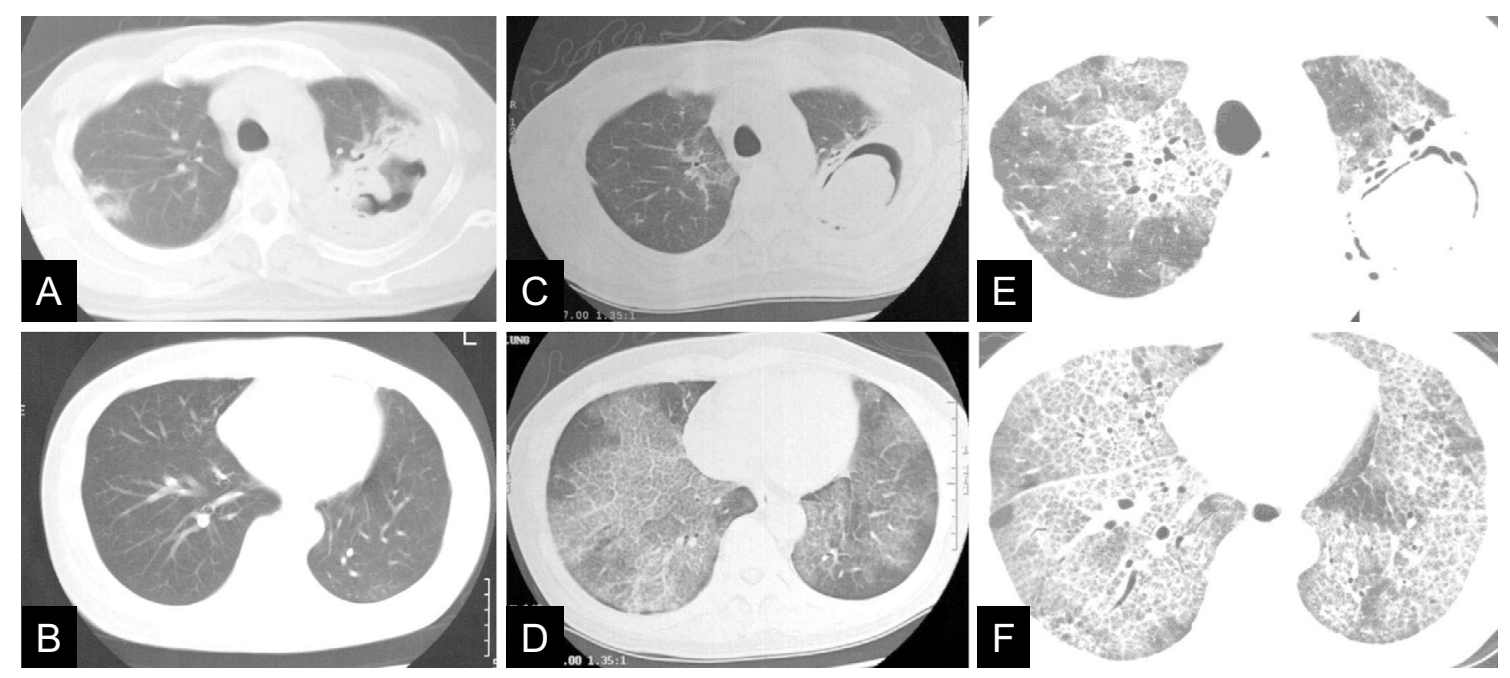

Figure 2. The chest CT scan. A thick-walled cavity was observed in the left upper lobe (A), and ground-glass opacity (GGO) was not observed at the diagnosis of Aspergillus infection (A, B). At the diagnosis of autoimmune pulmonary alveolar proteinosis (APAP), GGO suggestive of APAP appeared mainly in the lower lung field (D), and a fungus ball was present in the cavity (C). GGO with a crazy paving pattern extended to all lung fields, and the fungus ball enlarged in the cavity at commencement of therapy using granulocyte-macrophage colony-stimulating factor (E, F).

lavage appeared milky. Transbronchial lung biopsy specimens revealed alveolar wall thickening, alveolar type II epithelial cell hyperplasia, and periodic acid-Schiff stain (PAS)positive microgranular materials in the alveolar spaces. The patient's serum was positive for anti-GM-CSF autoantibody (90.3 $\mu \mathrm{g} / \mathrm{mL}$, normal level: $<0.5 \mu \mathrm{g} / \mathrm{mL})$. The serum levels of Krebs von den Lungen-6 (KL-6) and surfactant protein-D (SP-D) were elevated to $5,700 \mathrm{U} / \mathrm{mL}$ and $583 \mathrm{ng} / \mathrm{mL}$, respectively (normal levels: $<500 \mathrm{U} / \mathrm{mL}$ and $110 \mathrm{ng} / \mathrm{mL}$, respectively). The patient was subsequently diagnosed with APAP complicated by pulmonary aspergillosis.

Six months after the diagnosis of APAP (Fig. 1C, 2E, F), the patient's pulmonary function became severely disturbed, with a percentage predicted value of vital capacity and diffuse capacity of carbon monoxide at $39.5 \%$ and $20.4 \%$, re- spectively. An arterial blood gas analysis in the supine position under room air revealed a partial pressure of arterial oxygen $\left(\mathrm{PaO}_{2}\right)$ of 36.8 Torr. The disease severity of APAP was grade $\mathrm{V}$, as defined by Inoue et al. (11). The serum KL6 and SP-D levels were elevated to $24,500 \mathrm{U} / \mathrm{mL}$ and 1,180 $\mathrm{ng} / \mathrm{mL}$, respectively. Whole-lung lavage was deemed to be a contraindication due to active Aspergillus infection. The patient was treated by daily inhalation of $250 \mu \mathrm{g}$ of GM-CSF for eight weeks; however, his hypoxemia did not improve. Although a daily subcutaneous injection of $250 \mu \mathrm{g}$ of GMCSF for three weeks was added to the treatment regimen, hypoxia and radiological findings did not improve. The intravenous administration of micafungin (150 mg/day) and inhalation of amphotericin B (5 mg/body) were initiated as antifungal therapy in place of itraconazole, which had been 

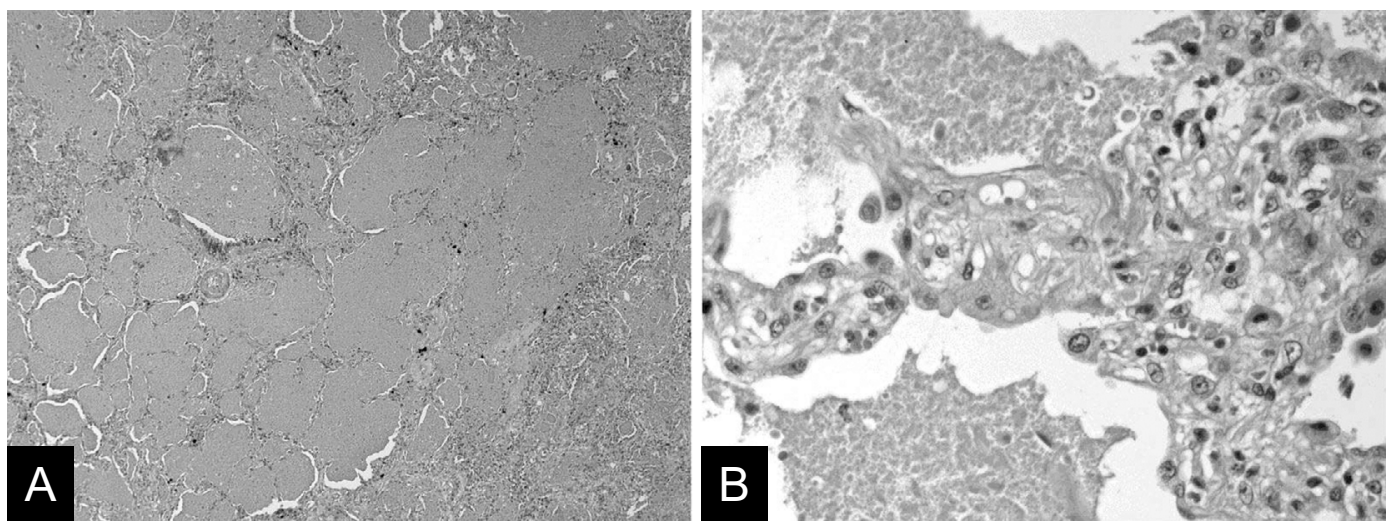

Figure 3. Pathological findings of necropsy specimens of the lung. The alveolar spaces were filled with PAS-positive microgranular material (A, B), and alveolar walls were lined with hyperplastic type II epithelial cells (B) (Hematoxylin and Eosin staining).

administered since the initial diagnosis of aspergillosis. The patient died of respiratory failure four months after the commencement of GM-CSF therapy.

Necropsy lung specimens revealed an intra-alveolar accumulation of PAS-positive material consistent with APAP, as well as neutrophil infiltration around bronchioles consistent with infectious bronchiolitis (Fig. 3); however, A. fumigatus was not detected in the small specimens. Additionally, interstitial pneumonia was not observed in the specimens. Hence, the chief cause of death was speculated to be a progression of APAP rather than Aspergillus infection.

\section{Discussion}

Immunodeficiency associated with APAP causes pulmonary aspergillosis $(4,5)$; however, in some cases, aspergillosis precedes APAP. Kobayashi et al. (9) reported that APAP in one patient following the onset of simple pulmonary aspergilloma resolved spontaneously after resection of the fungus ball. The resolution of PAP after the treatment of complicated rhinocerebral aspergillosis has also been reported (12). Hence, the pathophysiological association between Aspergillus infection and APAP is supported by these previous studies and aspergillosis may have affected the onset and progression of APAP in the present case.

The culture supernatant of aspergilli has been reported to suppress the macrophage function (13-15). Thus, aspergillosis could increase the deposition of microgranular materials in alveolar spaces, thereby worsening APAP by impairing phagocytosis by alveolar macrophages. The inhibition of macrophage function may be also associated with the disease onset of APAP. Anti-GM-CSF autoantibody is a pathogenic factor in APAP (16). However, low levels of anti-GMCSF neutralizing antibody have also been reported to be present in the serum of healthy subjects and multiple myeloid functions may be regulated by GM-CSF concentrations (17). Therefore, in some situations, the elevation of serum levels of anti-GM-CSF neutralizing antibody to a critical threshold can lead to the development of APAP (17). As- pergillus infection may lower the critical threshold for the concentration of anti-GM-CSF neutralizing antibody through its inhibitory effects on the macrophage function.

The production of anti-GM-CSF antibody may be stimulated in patients with Aspergillus infections. Th2 cells are activated in an animal model of aspergillosis $(18,19)$. This Th2 response is thought to be associated with autoimmune diseases by promoting the production of autoantibodies (20). Thus, Aspergillus infection may be an initiating factor for APAP in some cases.

The serum concentration of SP-D, which is reported to correlate with APAP severity $(4,21)$, has been found to increase in an in vivo murine model of aspergillosis (22) and in patients with allergic bronchopulmonary aspergillosis (23). The elevation of serum SP-D levels may reflect SP$\mathrm{D}$ overproduction by alveolar epithelial cells, as aspergilli stimulate SP-D production by type II alveolar epithelial cells in vitro (18). Therefore, Aspergillus infection may also be a promoting factor for APAP by stimulating SP-D production by type II alveolar epithelial cells.

The tolerance to GM-CSF therapy observed in the present case may be due to variable effects of aspergilli. The resection of the fungus ball in the left upper lobe in this case may have reduced the severity of APAP, as previously described in the reports of Kobayashi et al. (9) and Lin et al. (12). It may also have improved the response to GMCSF therapy. However, it was difficult to decide whether to perform resection of aspergilloma in the present case due to the progressive respiratory failure.

The hypothesis described above should be confirmed by clinical findings in other cases; however, it might be better that we suspect the complication of APAP if new groundglass opacity (GGO) developed on chest CT in cases of Aspergillus infection. Whether anti-GM-CSF antibody is present in the serum of patients with Aspergillus infection and whether the change in anti-GM-CSF antibody level is associated with other clinical findings suggesting the onset of APAP should be clarified. These findings together may help to reveal the relationship between APAP and Aspergillus in- 
fection. Furthermore, it may lead to improved methods for inducing remission of APAP cases that have become complicated due to Aspergillus infection and are resistant to GMCSF treatment.

Author's disclosure of potential Conflicts of Interest (COI). Yoshikazu Inoue: Honoraria, Shionogi \& Co., Ltd.

\section{Financial Support}

This study is partly supported by a grant from the Ministry of Health, Labour and Welfare, Japan \{H26-Itaku (Nan)-Ippan$077\}$.

\section{Acknowledgement}

We thank Dr. Yuko Takehara (Chubu-Rosai Hospital, Japan) for her valuable assistance with the treatment management and necropsy preparation.

\section{References}

1. Rosen SG, Castleman B, Liebow AA. Pulmonary alveolar proteinosis. N Engl J Med 258: 1123-1142, 1958.

2. Kitamura T, Tanaka N, Watanabe J, et al. Idiopathic pulmonary alveolar proteinosis as an autoimmune disease with neutralizing antibody against granulocyte/macrophage colony-stimulating factor. J Exp Med 190: 875-880, 1999.

3. Uchida K, Beck DC, Yamamoto T, et al. GM-CSF autoantibodies and neutrophil dysfunction in pulmonary alveolar proteinosis. $\mathrm{N}$ Engl J Med 356: 567-579, 2007.

4. Inoue Y, Trapnell BC, Tazawa R, et al. Characteristics of a large cohort of patients with autoimmune pulmonary alveolar proteinosis in Japan. Am J Respir Crit Care Med 177: 752-762, 2008.

5. Punatar AD, Kunse S, Blair JE, et al. Opportunistic infections in patients with pulmonary alveolar proteinosis. J Infect 65: 173-179, 2012.

6. Pascual J, Gomez Aguinaga MA, Vidal R, et al. Alveolar proteinosis and nocardiosis: a patient treated by bronchopulmonary lavage. Postgraduate Medical J 65: 674-677, 1989.

7. Witty LA, Tapson VF, Piantadosi CA. Isolation of mycobacteria in patients with pulmonary alveolar proteinosis. Medicine (Baltimore) 73: 103-109, 1994.

8. Matsuhisa T, Mori T, Urata A, et al. Pulmonary alveolar proteinosis exacerbated by aspergilloma. J Jpn Soc Respir Endoscopy 27: 372-377, 2005.

9. Kobayashi T, Ebina M, Oshima M, et al. Case of alveolar proteinosis associated with pulmonary aspergillosis. Nihon Naika Gak- kai Zasshi (The Journal of the Japanese Society of Internal Medicine) 88: 135-137, 1999 (in Japanese).

10. Izumikawa $\mathrm{K}$, Tashiro $T$, Tashiro $M$, et al. Pathogenesis and clinical features of chronic pulmonary aspergillosis - is it possible to distinguish CNPA and CCPA clinically? J Infect Chemother 20: 208-212, 2014.

11. Inoue $Y$, Nakata $K$, Arai $T$, et al. Epidemiological and clinical features of idiopathic pulmonary alveolar proteinosis in Japan. Respirology 11: S55-S60, 2006.

12. Lin KP, Sheng WH, Wang CP, et al. Resolution of secondary pulmonary alveolar proteinosis following treatment of rhinocerebral aspergillosis. Int J Infect Dis 14 (Suppl 3): e246-e249, 2010.

13. Murayama T, Amitani R, Ikegami Y, et al. Suppressive effects of Aspergillus fumigatus culture filtrates on human alveolar macrophages and polymorphonuclear leucocytes. Eur Respir J 9: 293300, 1996.

14. Murayama T, Amitani R, Ikegami Y, et al. Effects of Aspergillus fumigatus culture filtrate on antifungal activity of human phagocytes in vitro. Thorax 53: 975-978, 1998.

15. Kamei K, Watanabe A, Nishimura K, et al. Cytotoxicity of Aspergillus fumigatus culture filtrate against macrophages. Nippon Ishinkin Gakkai Zasshi (Japanese Journal of Medical Mycology) 43: 37-41, 2002 (in Japanese, Abstract in English).

16. Sakagami T, Uchida K, Suzuki T, et al. Human GM-CSF autoantibody and reproduction of pulmonary alveolar proteinosis. N Engl J Med 361: 2679-2681, 2009.

17. Uchida K, Nakata K, Suzuki T, et al. Granulocyte/macrophage colony stimulating factor autoantibodies and myeloid cell immune functions in healthy subjects. Blood 113: 2547-2556, 2009.

18. Haczku A, Cao Y, Vass G, et al. IL-4 and IL-13 forms a negative feedback circuit with surfactant protein-D in the allergic airway response. J Immunol 176: 3557-3565, 2006.

19. Madan T, Kishore U, Singh M, et al. Surfactant protein A and D protected mice against pulmonary hypersensitivity induced by Aspergillus fumigatus antigen and allergens. J Clin Invest 107: 467475, 2001.

20. Fairweather D, Frisancho-Kiss S, Rose NR. Sex difference in autoimmune disease from a pathological perspective. Am J Pathol 173: 600-609, 2008.

21. Arai T, Inoue $Y$, Sugimoto $C$, et al. CYFRA 21-1 as a disease severity marker for autoimmune pulmonary alveolar proteinosis. Respirology 19: 246-252, 2014.

22. Atochina EN, Beers MF, Tomer $\mathrm{Y}$, et al. Attenuated allergic airway hyperresponsiveness in C57BL/6 mice is associated with enhanced surfactant protein (SP)-D production following allergic sensitization. Respir Res 4: 15, 2003.

23. Krane M, Griese M. Surfactant protein D in serum from patients with bronchopulmonary aspergillosis. Eur Respir J 22: 592-595, 2003.

(C) 2015 The Japanese Society of Internal Medicine http://www.naika.or.jp/imonline/index.html 